\title{
PLANTAS DE IMPORTANCIA ECONÓMICA Y ECOLÓGICA EN EL JARDÍN BOTÁNICO - ARBORETUM EL HUAYO, IQUITOS, PERÚ
}

\author{
Heiter Valderrama Freyre ${ }^{1}$
}

\begin{abstract}
RESUMEN
La Universidad Nacional de la Amazonía Peruana, a través de la Facultad de Ingeniería Forestal, está desarrollando el Jardín Botánico - Arboretum El Huayo (JBAH), con la finalidad de mostrar a la comunidad nacional e internacional las experiencias de 29 años de conservación, in situ y ex situ, de la diversidad biológica. El JBAH se localiza en un área de 1300 ha de bosques, de las que una parte son bosques naturales con diferentes ecologías, y otra son plantaciones de diferentes especies y edades. La población humana existente en el área de influencia del JBAH utiliza diversas partes de numerosas especies vegetales para solucionar, en parte, los problemas básicos de la comunidad. De igual modo, existen especies vegetales de importancia ecológica en el área, las que con un adecuado manejo garantizarán la permanencia de las características del ecosistema forestal. En el presente estudio, que se realizó en el marco del proyecto Diversidad Biológica de la Amazonía Peruana, Perú - Finlandia (BIODAMAZ), a través de encuestas realizadas a la población involucrada en el área de influencia del JBAH, y con ayuda de material bibliográfico, se publica una relación de 256 especies vegetales usadas, de las cuales el $26.13 \%$ corresponde a especies que se usan como madera redonda para la construcción de viviendas rurales, el $24.77 \%$ se usa como madera aserrada, el $13.86 \%$ son especies de uso medicinal, y el $13.41 \%$ de uso alimentario. Por su importancia económica y porque algunas de estas especies son de distribución restringida, merecen una mayor atención en el programa de conservación ex situ e in situ del JBAH. Es necesario elaborar planes de manejo y el enriquecimiento de los bosques con las especies utilizadas, para garantizar el aprovechamiento sustentable de estos recursos.
\end{abstract}

Palabras clave: Amazonía peruana, especies vegetales utilizadas, importancia ecológica, Jardín Botánico Arboretum El Huayo, madera aserrada, madera redonda, uso alimenticio, uso medicinal.

\begin{abstract}
The Faculty of Forest Engineering of the Peruvian Amazonian National University is developing the El Huayo Botanical Garden-Arboretum (JBAH), with the aim of showing the national and international community the experience of 29 years of in situ and ex situ conservation of biological diversity. The JBAH is located in a forestal area of 1,300 ha, made up in one part of different types of natural forest and, in another part, of plantations of different species of varying ages. The human population living in and around the area of the JBAH uses numerous plant species to solve some of their communities' basic problems. Furthermore, there are ecologically important plant species in the area that, with adequate management, will guarantee the existence of the forest ecosystem. In the present study, conducted as part of the Peru-Finland Biological Diversity of the Peruvian Amazon project (BIODAMAZ), using questionnaires among the population involved in the JBAH intervention area, and with the help of bibliographic material, we publish a list of 256 plant species. Of these species, $26.13 \%$ are used as round wood for construction of rural houses, $24.77 \%$ is used as sawn wood, $13.86 \%$ is used in traditional medicine and $13.41 \%$ can be used as food stuffs. Because of their economic importance and because some of these species have a restricted distribution, they deserve special attention under the JBAH in situ and ex situ conservation programme. It is important to develop management and forest enrichment plans for the species used, so as to guarantee the sustainability of their use in the area.
\end{abstract}

Key words: Botanical Garden-Arboretum El Huayo, construction wood, ecological importance, edible plants, medicinal plants, Peruvian Amazon, timber, usable plants.

1 Proyecto Diversidad Biológica de la Amazonía Peruana, Perú-Finlandia (BIODAMAZ). Av. Abelardo Quiñones, km 2.5, Iquitos, Perú. Correo electrónico: biodamaz@iiap.org.pe / Facultad de Ingeniería Forestal, Universidad Nacional de la Amazonía Peruana (UNAP), Iquitos, Perú. 


\section{INTRODUCCIÓN}

La población asentada tanto en el bajo Nanay y el área de influencia de la carretera Iquitos - Nauta como en el área de influencia del Jardín Botánico-Arboretum El Huayo (JBAH) de la Universidad Nacional de la Amazonía Peruana (UNAP), está utilizando muchas especies forestales y plantas existentes en los bosques naturales para satisfacer sus necesidades básicas. Sin embargo, estos recursos no constituyen una base importante para el desarrollo industrial ni contribuyen por tanto a mejorar significativamente su calidad de vida.

Son necesarios programas de capacitación y extensión para enseñar a las comunidades locales a conservar, manejar y usar en forma sustentable los recursos naturales existente en estos bosques, hoy amenazados por la sobre explotación y la agricultura migratoria, de modo que rindan beneficios de forma sostenida y contribuyan a su desarrollo humano integral.

El presente trabajo ha sido realizado en del marco del proyecto Diversidad Biológica de la Amazonía Peruana, Perú - Finlandia (BIODAMAZ) ${ }^{2}$, en coordinación con la Facultad de Ingeniería Forestal (FIF) de la UNAP. Presentamos aquí la relación de las especies forestales y plantas del Arboretum y otras parcelas del JBAH. Éstas han sido categorizadas por usos locales (según información de los mismos pobladores), y por usos de la madera en la industria forestal, especialmente con respecto al comportamiento al aserrío, trabajabilidad, secado, durabilidad natural, preservado, y resistencia mecánica. También presentamos una clasificación preliminar de los usos probables de la madera calificados a nivel de laboratorio, junto con una comparación de resultados existentes sobre especies tropicales, basado en la literatura existente. Finalmente, se citan especies cuyos productos diferentes a la madera son usados por la industria, y especies de importancia ecológica y/o científica.

En resumen, este estudio ha tenido como objetivo determinar las especies de importancia económica y ecológica, existentes en el área de influencia del JBAH y realizar la clasificación de uso de los productos maderables, y diferentes a la madera, usados por la comunidad del área de influencia del JBAH.

\section{ANTECEDENTES}

Según la información registrada en el Mapa de Tipos de Bosques del Fundo UNAP, del total de 2001.10 ha, 1 121.64 ha (equivalente al 56.85\%) corresponden a la clasificación de bosques intervenidos y bosques de terrazas medias; gran parte de esta área le corresponde al JBAH. (BIODAMAZ, 2004; ver mapa en el Sistema de Información del Jardín Botánico-Arboretum El Huayo - SIHUAYO, www.siamazonia.org.pe). Estos bosques intervenidos son el producto de la eliminación del bosque primario, debido principalmente a la agricultura migratoria. Los bosques de terrazas medias se caracterizan porque ocupan una posición más alta que los bosques de terrazas bajas y no están sujetos a inundaciones periódicas habituales, sino solo a inundaciones de carácter excepcional. Presentan una fisiografía de relieves planos o ligeramente inclinados.

Una gran parte de los bosques del JBAH se encuentra actualmente en diferentes estadíos de regeneración, por haber sido abandonados después de unos años de uso agrícola o maderero.

Dourojeanni (1981), manifiesta que la mayor parte de los bosques secundarios o "purmas" de la Amazonía peruana son, como en cualquier otra región del trópico húmedo, barbechos forestales, es decir, la consecuencia de la agricultura migratoria. Los suelos existentes en estas áreas, de baja fertilidad relativa, son abandonados o dejados en descanso por un determinado período de tiempo, dando origen al bosque secundario, purma o barbecho forestal. Las especies que se desarrollan en el bosque secundario son intensamente utilizadas en el Perú. Entre las formas de uso industrial y tradicional se pueden mencionar: la industria forestal (pulpa, aserrío, cajonería), construcciones rurales, artesanía, combustible, medicina, plantas ornamentales, y como alimento humano y animal.

2 Proyecto Diversidad Biológica de la Amazonía Peruana (BIODAMAZ) es un convenio entre los gobiernos del Perú y de Finlandia ejecutado conjuntamente por el Instituto de Investigaciones de la Amazonía Peruana (IIAP) y por el consorcio finlandés formado por la empresa de consultorías ambientales, Biota BD Oy, y por la Universidad de Turku. 
Sin embargo, pese a que los pobladores rurales de la Amazonía están utilizando muchas especies forestales de los bosques secundarios para satisfacer sus necesidades básicas, estos recursos no están apoyando el desarrollo industrial ni contribuyen a mejorar significativamente su calidad de vida. Para lograr esto se necesita impulsar la organización y la capacitación de los pobladores locales en tecnologías de uso sostenible del bosque, como alternativa a la agricultura migratoria. Los bosques secundarios en esta zona están conformada en su mayor porcentaje por "topa" (Ochroma lagopus), "cético" (Cecropia sp.), Schizolobium parahybum, y "huamanzamana" (Jacaranda copaia (Aubl.) D. Don); entre otras especies. Estos árboles se caracterizan por un crecimiento rápido, vertical, con un solo tallo principal, con hojas compuestas o lobuladas, grandes, simples y ramas bajas deciduas.

Nosotros podemos clasificar las más importantes plantas utilizadas como: (a) plantas que se usan para producir aceites y ceras, 35 especies; (b) plantas para obtener aromas y cosméticos, 37 especies; (c) plantas comestibles de nuestra selva (plantas nativas no introducidas), 524 especies, de las que se consume las raíces, cogollos, hojas, flores o frutos (en Iquitos se conocen 182 especies de plantas silvestres que producen frutos comestibles, y el 80\% de éstas es comercializado en el mercado local); (d) para madera 401 especies; (e) para latex 21 especies; (f) para colorantes y tintes naturales 22 especies; (g) plantas que producen tóxicos, 334 especies; y (h) plantas medicinales conocidas y utilizadas en nuestra selva, 3213 especies.

Con respecto al grupo de especies utilizadas como madera redonda para la construcción de viviendas, Soto y Vásquez (1989), presentan la relación de 29 especies. Estos autores indican que se debe realizar una clasificación preliminar en madera de primera, segunda y tercera (ver abajo en Clasificación de acuerdo a usos locales y Resultados y Discusión), categorizada de acuerdo al uso específico dentro de la estructura de la vivienda, llámese horcones (columnas), vigas, soleras, caibros, etc.; cada uso específico requiere ciertas características en calidad y tamaño (longitud y diámetro, etc.). Además, esta clasificación debe ceñirse técnicamente a ciertas características tecnológicas y organolépticas, y a propiedades de la madera, como inferencia en la durabilidad natural, abundancia de células parenquimatosas observadas a nivel macroscópico, densidad, porcentaje de duramen, entre otras. De igual manera, autores como Lao y Flores (1972), Encarnación (1983), y Vásquez (1989), describen numerosas especies forestales utilizadas localmente por las comunidades de la zona como madera redonda para la construcción de viviendas (horcones, caibros, soleras, vigas, etc.), combustible, artesanía (madera, flor, fruto, etc.), amarres de todo tipo, tintes, construcción de canoas y/o embarcaciones, comestibles (frutos), uso medicinal y ornamental, entre otros.

Del mismo modo, Bueno (1970), manifiesta que existen muchas especies que crecen en purmas y que son utilizadas en la industria forestal. Los géneros Cecropia, Ficus, Hura, Guazuma, Inga, y Jacaranda, por ejemplo, son empleados en la fabricación de pulpa y papel. También se está utilizando especies del bosque secundario para la industria del aserrío como "topa" (Ochroma pyramidale (Cav. ex Lam) Urban), y los géneros Gauzuma, Schizolobium, Inga, y Albizzia, para diferentes usos según las características tecnológicas que posee la madera.

Gracias a la destacada labor de muchos investigadores se dispone hoy de información sobre las propiedades físicas y mecánicas de la madera de especies comunes existentes en purmas y bosques secundarios, así como de sus usos actuales y potenciales. Entre ellos podemos citar a Aróstegui (1974; 1975; 1979; 1982), Aróstegui y Sato (1970), Aróstegui y Sobral (1986), Aróstegui y Valderrama (1986), Valderrama y Torres (1998), JUNAC (1981b), y Valderrama (1984; 1993). Cabe destacar también los estudios sobre estructura anatómica de la madera de muchas especies del trópico amazónico y de algunas especies que se desarrollan en purmas, entre ellos Begazo y Aróstegui (1985), JUNAC (1981a), y Valderrama et al. (1989). Así mismo ITTO (1991), y Valderrama (1992), entre otros, determinaron el comportamiento tecnológico de la madera y de una u otra forma recomiendan los usos probables de la madera de numerosas especies de árboles amazónicos. 


\section{MATERIAL Y MÉTODO}

\section{1. Área de estudio}

El estudio se realizó con especies vegetales identificadas en el Jardín Botánico-Arboretum El Huayo (JBAH), ubicado en el Centro de Investigación y Enseñanza Forestal Puerto Almendra (CIEFOR) de la Facultad de Ingeniería Forestal (FIF) de la Universidad Nacional de la Amazonía Peruana (UNAP). El JBAH se localiza en la margen derecha del río Nanay, afluente del río Amazonas, provincia de Maynas, departamento de Loreto. Se localiza aproximadamente en las siguientes coordenadas: $3^{\circ} 49^{\prime} \mathrm{S}, 7^{\circ} 25^{\prime}$ O. (BIODAMAZ, 2004; ver mapa del JBAH en SIHUAYO, www.siamazonia.org.pe).

El área de evaluación y de muestreo, corresponden a las primeras cinco parcelas del Arboretum El Huayo, cada parcela tiene 1.18 ha, sumando en total 5.90 ha, con árboles de $10 \mathrm{~cm}$ a más de DAP (diámetro en la altura de pecho), inventariadas al 100\%, del mismo modo, se evaluaron especies existente fuera de la jurisdicción del Arboretum, pertenecientes a otros componentes del JBAH.

\subsection{Métodos}

Los usos locales fueron determinados a través de encuestas y conversaciones con pobladores oriundos del lugar, en este caso pertenecientes a los caseríos de Puerto Almendra y Nina Rumi, ambos dentro de la jurisdicción del JBAH. Las identificaciones botánicas fueron realizadas conforme a metodologías estandarizadas.

Las especies fueron elegidas de acuerdo al inventario, realizado con la finalidad de estudiar la composición florística, indicar la abundancia y analizar el gregarismo de la vegetación en cada parcela del Arboretum. Paralelamente al inventario florístico, fueron colectadas muestras botánicas (hojas, flores y frutos) de los árboles seleccionados, las cuales fueron identificadas en colaboración con el Herbarium Amazonense (AMAZ) de la UNAP. Los estudios de los aspectos ecológicos y fitosociológicos de las primeras cinco parcelas del Arboretum fueron realizados por Valderrama (1998; 2000a; 2000b; 2000c; 2000d). En estos trabajos podemos encontrar las especies de mayor importancia ecológica en el área, y una descripción de la estructura horizontal y vertical de la vegetación. La relación de especies seleccionadas por su importancia económica y ecológica se presenta en el Cuadro 1. Una relación completa de las especies forestales seleccionadas y clasificadas de acuerdo a su importancia económica y ecológica es accesible a través de SIHUAYO (www.siamazonia.org.pe).

\section{Clasificación de acuerdo a usos locales}

Las especies fueron clasificadas de acuerdo a sus usos locales, teniendo en consideración no sólo su valor económico sino científico y ecológico. De acuerdo con estos criterios, las especies fueron clasificadas en los siguientes grupos:

Madera redonda para la construcción de viviendas

Se refiere a aquellas utilizadas en las viviendas de tipo rústico, como vigas, soleras, techos, pisos, etc. La madera es talada con diámetros de $10 \mathrm{~cm}$ a $35 \mathrm{~cm}$, aproximadamente. La gente clasifica como maderas "de primera" aquéllas de mayor valor, es decir las que tienen mayor durabilidad natural en condiciones adversas de uso, son más densas y no son atacadas por insectos; las maderas de segunda, en cambio, son más suaves, de menos durabilidad natural y son fácilmente atacadas por agentes biológicos; éstas se usan solamente bajo sombra (Soto y Vásquez, 1989).

Madera aserrada

Las especies usadas como madera aserrada son aquéllas de las que se fabrican tablas, tablones, listones y otros. La bibliografía nos permite clasificar estas maderas de acuerdo a los diferentes comportamientos tecnológicos durante el aserrío, la durabilidad natural, el secado, el preservado, la resistencia mecánica y la trabajabilidad. De igual manera, se ha clasificado a la madera aserrada de acuerdo a usos probables para construcción de 
viviendas (estructuras como columnas y vigas, carpintería de obra como puertas, ventanas, pisos y parquet), encofrados, construcción pesada, mueblería, laminado, chapas decorativas, mangos de herramientas, carrocerías, cajonería pesada, cajonería liviana, artesanía, fabricación de pulpa y papel, y embarcaciones. Para la determinación de estos usos se ha utilizado información obtenida en el Laboratorio de Anatomía y Tecnología de la Madera de la FIF de la UNAP. Entre otros autores podemos citar: Aróstegui (1974; 1975; 1979; 1982); Aróstegui y Valderrama (1986); Valderrama (1984; 1992; 1993); Valderrama y Torres (1998).

\section{Uso alimenticio}

Incluye plantas de las que se aprovecha frutos y semillas, así como chonta (palmito) y otros. Se basa principalmente en la información recopilada por los pobladores de la zona. Fueron seleccionadas especies importantes por sus frutos y otras partes del árbol, comestibles para el hombre, así como para la fauna silvestre terrestre y acuática.

\section{Uso ornamental}

Incluye especies seleccionadas por su atractivo y aspecto decorativo (floración, forma de copa, etc.), de rápido crecimiento y con ventajas comparativas para su tratamiento silvicultural.

\section{Artesanía}

Incluye especies seleccionadas por su uso local en la artesanía. Las partes de la planta usadas con este fin de acuerdo con los pobladores son: ramas, frutos, yemas terminales, cortezas, tallos, lianas, madera del fuste, raíces, semillas, y productos extractivo (tintes y resinas).

Medicinal

Son plantas usadas en la medicina tradicional amazónica. De acuerdo a la información proporcionada por la población local, fueron seleccionados árboles y plantas que difieren en las partes utilizadas, forma de aplicación, etc. Parte de la información fue recogida en fuentes bibliográficas (Vargas, 1996).

Uso industrial

Con base en la información recogida entre la población local, fueron seleccionadas especies con potencial de uso industrial diferente a la madera, como cortezas, latex, chonta, frutos, fibras, tintes, aceites, semillas, etc.

Especies de importancia ecológica

Sobre la base de los resultados obtenidos por Valderrama (1998; 2000a; 2000b; 2000c; 2000d), se ha podido determinar las especies de mayor y menor importancia ecológica existentes en el JBAH. La existencia de estas especies garantizaría la permanencia de la vegetación característica de la zona.

\section{Importancia ecológica}

Mayor valor ecológico

Especies categorizadas de acuerdo al mayor valor o importancia ecológica y que existen en las cinco primeras parcelas del Arboretum.

Raras o de distribución restringida

Incluye especies cuyo número de individuos por especies es muy restringido y que existen en las cinco primeras parcelas del Arboretum.

\section{RESULTADOS Y DISCUSIÓN}

Una relación completa de las especies forestales seleccionadas y clasificadas de acuerdo a su importancia económica y ecológica es accesible a través de SIHUAYO (www.siamazonia.org.pe). Todos los cuadros referidos están disponibles en SIHUAYO para mayor información. El presente artículo incluye el Anexo 1. 
En el Anexo 1 se presenta el listado de 256 especies priorizadas por su importancia económica, científica y ecológica. La mayoría se encuentra en el área de influencia del JBAH, como "sangre de grado" (Croton lechleri Muell. Arg.), entre otras especies. Existen especies que tienen dos o más usos locales como "sacha casho" (Anacardium giganteum Hancock), "palo de rosa" (Aniba rosaeodora Ducke), y "copaíba" (Copaifera paupera (Harns) S.). Estas especies son de distribución restringida actualmente, debido a la presión extractiva que por su uso económico han sufrido históricamente estas especies, particularmente el "palo de rosa". Hoy estas especies son bastante raras, por lo que merecen una mayor atención durante el programa de conservación ex situ dentro del JBAH. Existe un número mayor de especies que son usadas para aserrío y como madera redonda para construcción; esto implica la necesidad de elaborar planes de manejo y enriquecimiento de los bosques con estas especies, para garantizar su aprovechamiento sostenible.

Considerando que la mayoría de especies tienen uno o más usos locales alternativos, en la Figura 1 podemos observar que del total de especies seleccionados, el $26.13 \%$ le corresponde a especies que se usan como madera redonda para la construcción de viviendas rurales, el $24.77 \%$ se usa como madera aserrada, el $13.86 \%$ son especies de uso medicinal, y el $13.41 \%$ de uso alimenticio.

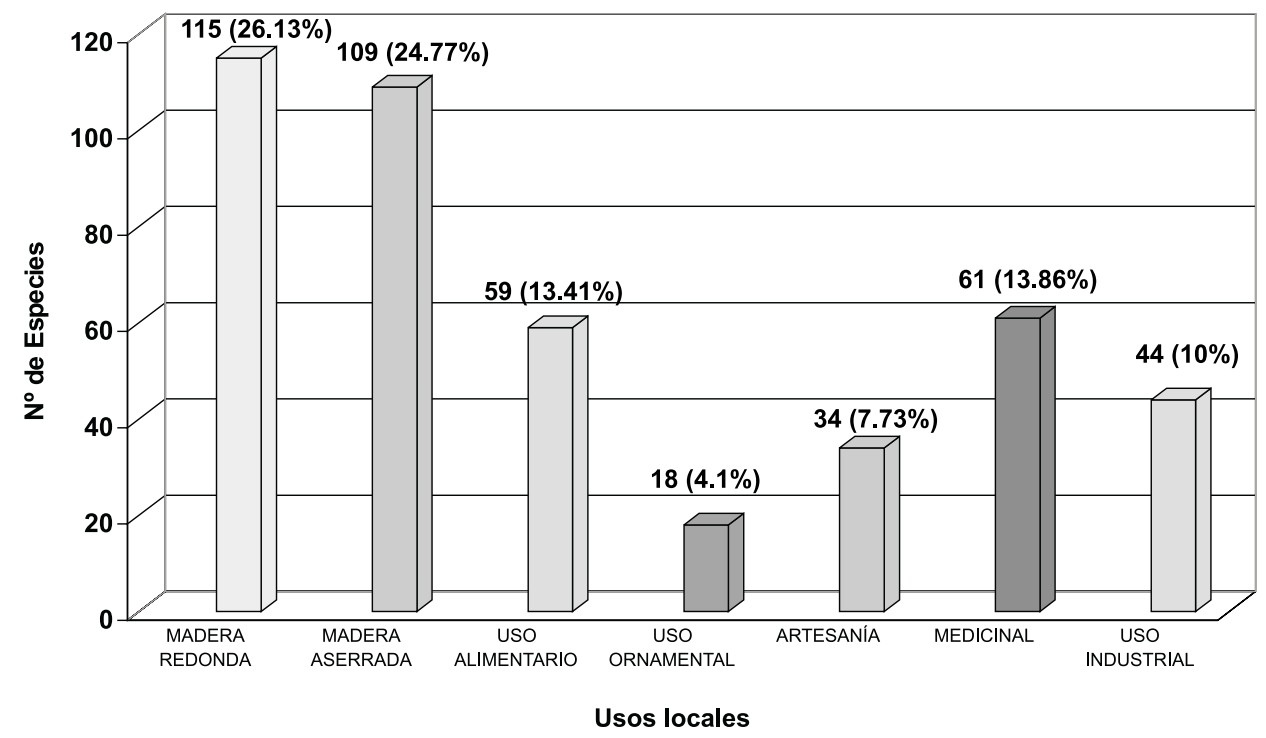

Figura 1. Representación gráfica de las especies por usos locales.

Los criterios tecnológicos y las propiedades de la madera, juntamente con las dimensiones (diámetro, longitud) de los miembros estructurales, constituyen la base para la categorización de las especies por usos específicos como madera redonda: horcones (columnas), vigas, soleras, caibros, etc. Se identifica 115 especies (Anexo 1; ver el Cuadro 2 en SIHUAYO, www.siamazonia.org.pe), que se usan como madera redonda para la construcción de viviendas rústicas en la zona. Los árboles destacan en altura, y son talados a los 5 ó 10 años por moradores. Por otro lado, existen extractores que en forma ilegal penetran dentro del área de influencia del JBAH, talan estas maderas y las comercializan en la localidad de Iquitos. Destacan para este uso especies de las familias Lauraceae, Myristicaceae, Annonaceae, Burseraceae y Meliaceae, entre otras. De igual modo, destacan especies de los géneros: Aniba ("moena"), Caraipa ("aceite caspi"), Eschweilera ("machimango"), Guarea ("requia"), Guatteria ("carahuasca"), Iryanthera ("cumala colorada"), Licania ("parinari”), Miconia ("rifari”), Protium (“copal"), Sloanea (“cepanchina"), y Virola (“cumala").

En este grupo la madera se clasifica de acuerdo a su uso dentro de la estructura de la vivienda, pudiendo ser para vigas, soleras, caibros, columnas, ishpaneras, etc. La comercialización se realiza considerando una clasificación preliminar en las siguientes categorías: madera de primera (aquélla de densidad media, alta y muy alta, durabilidad 
natural buena en condiciones adversas de uso, duramen pronunciado); madera de segunda (densidad baja a media, durabilidad natural de media a baja, albura pronunciada). Existen especies que son potencialmente usadas como columnas, destacando: "añuje moena" (Anaueria brasiliensis Kosterm.), "pumaquiro" (Aspidospema macrocarpon Mart.), "parinari” (Licania macrocarpa Cuatre.), "caimito" (Pouteria guianensis Aubl.), "huacapú" (Minquartia guianensis Aublet.), "azufre caspi" (Moronobea coccinea Aubl.), y "machimango" Eschweilera coriacea ((A,D,C) S. Mori.). Existen grupos de especies que son considerados como madera de segunda, pese a que tienen densidad alta, duramen pronunciado y están exentas de albura, pero son susceptibles al ataque de agentes biológicos. Éstas son utilizadas por las comunidades como horcones en forma de "madera labrada", en algunos casos con buenos resultados ("machimango"). Un aspecto importante a tener en cuenta, que es considerado como el "cuello de botella" en la utilización de este producto, es el área de contacto directo con el suelo, donde tiende a pudrirse más rápidamente. Existen metodologías que se debería aplicar a fin de prolongar la vida útil de la madera.

De igual manera las especies que en forma común se usan para caibros y soleras son: "canela moena" (Anaueria brasiliensis Kosterm.), “aceite caspi” (Caraipa tereticaulis Tulasne), "carahuasca” (Guatteria elata R.E. Fries), "espintana" (Oxandra xylopioides Diel), "copal” (Protium ferrugineum (Engler) E.), "quillosisa" (Qualea paraensis Ducke), y "huira caspi” (Tapirira guianensis Aublet.).

Se presenta la relación de 97 especies que las comunidades usan como madera aserrada (Anexo 1; ver los Cuadros 3 y 4 en SIHUAYO, www.siamazonia.org.pe). Dentro de las especies que destacan en el uso para estructuras de viviendas podemos citar: "moena amarilla" (Aniba amazonica (Meiz) Mez), "lagarto caspi" (Calophyllum brasiliense Cambers), "andiroba" (Carapa guianensis Aubl), "tornillo" (Cedrelinga cateniformis Ducke), "copaíba" (Copaifera paupera (Harns) S.), y "quillosisa" (Vochysia vismiifolia Spruce ex Warming). Estas mismas especies se usan en la fabricación de puertas y ventanas. Para pisos y parquet destacan las especies: "moena amarilla", "mari mari" (Hymenolobium pulcherrimum Ducke), y "andiroba". En mueblería destacan las especies de "cedro" (Cedrela odorata L.), "tornillo", "copaíba”, y "caoba" (Swietenia macrophylla King).

Existen numerosas especies que carecen de estudios tecnológicos, y la información reportada en el presente documento, basada no sólo en información bibliográfica sino en encuestas a pobladores locales, servirá como referencia para orientar el comportamiento tecnológico y los usos definitivos de la madera.

El poblador amazónico, a través de muchas generaciones, orientó el uso de la madera para diferentes aplicaciones, como pisos, artesanía, pilotes, durmientes, postes, etc. Sin embargo, algunas especies poseen problemas de uso, y el desconocimiento de sus características y propiedades tecnológicas puede ser un limitante para mejorar la calidad del producto, originando el mal empleo de las piezas estructurales.

Existen grupos de especies aserrables como "añuje moena", "azúcar huayo" (Hymenaea spp.), "azufre caspi”, "carahuasca" (Guetteria spp.), "chontaquiro" (Swartzia cardiosperma Spruce ex Bentham), "cumala" (Osteophloeum platyspermun (A.DC.) W., Virola spp., Iryanthera spp.) "requia" (Trichilia spp.), "shicshi moena" (Ocotea oblonga (Mez) Rohwer), entre otras, que son utilizadas además como madera redonda para construcción de viviendas rurales, y que son comercializadas incluso en el mercado de la ciudad de Iquitos. Esta mala política de utilización de especies valiosas a ese nivel de crecimiento, origina una pérdida de rendimiento en función al volumen de madera aserrada que el bosque generaría posteriormente, por lo que sería conveniente utilizar como madera redonda sólo aquellas especies cuyo diámetro, en condición de árbol maduro, no alcancen lo requerido por la industria del aserrío.

Se enumera 59 especies (Anexo 1; ver el Cuadro 5 en SIHUAYO, www.siamazonia.org.pe), con frutos comestibles tanto para humanos como para la fauna silvestre terrestre y acuática. Muchas de ellas son conocidas a nivel del mercado local como: "aguaje" (Mauritia flexuosa L.F.), "almendro" (Caryocar spp.), "chambira"(Astrocaryum chambira Burret), "quinilla" (Chrysophyllum spp.), "huasaî" (Euterpe precatoria C. Martius), "parinari” (Licania spp.), "ubos" (Spondias mombin L.), "macambo" (Theobroma bicolor Humb), "balata rosada" (Micropholis guyanensis subesp. guayanensis), "ungurahui” (Oenocarpus bataua (Mart) Burret), "uvilla” (Pourouma spp.) y "humarí" (Poraqueiba sericea Tulasne). Es importante indicar las especies que los peces utilizan como alimento, como "ipururo" (Alchornea discolor E.P.) y "palometa huayo" (Neea parviflora (Poeppig \& Endlicher)). El 
manejo de las especies comestibles constituirá una alternativa de subsistencia para la población asentada dentro del área de influencia del JBAH. Muchas de estas especies se encuentran en estado natural en los bosques primarios y secundarios de la Amazonía.

Se presenta una relación de 18 especies (Anexo 1; ver el Cuadro 6 en SIHUAYO, www.siamazonia.org.pe), que la población usa como ornamentales y que constituyen una alternativa para la reforestación de la zona urbana de la ciudad. Se enumera 34 especies (Anexo 1; ver el Cuadro 7 en SIHUAYO, www.siamazonia.org.pe), que por sus usos locales pueden ser utilizadas en la industria de artesanías. Para ello la población utiliza su madera, en algunos casos el tallo completo, frutos, semillas, tintes que se extrae de la corteza, y raíces. La mayoría de estas especies son utilizadas en diferentes productos (canastos, bolsas, tejidos, juguetes, adornos, etc.), y son comercializadas en tiendas de la ciudad de Iquitos.

Se enumera 61 especies (Anexo 1; ver el Cuadro 8 en SIHUAYO, www.siamazonia.org.pe), que tienen diferentes usos y aplicaciones en la medicina tradicional local. Destacan especies que sirven para curaciones de enfermedades vaginales post-parto, reumatismo, malaria, úlceras, hernias, cicatrizantes, purgantes, antimicóticos, y para tratar otras enfermedades cutáneas, para desinflamar heridas, para tratamiento de la estomatitis (pátco en la boca del bebé), y para enfermedades renales, dolores y resfríos. Se indica la forma del preparado para su aplicación (maceración, cocción, emplasto, en forma directa, triturado, etc.), y la parte utilizada de la planta (hojas, corteza, látex, raíz, etc.).

Hay especies medicinales muy conocidas en Iquitos, entre ellos: "ipururo" (Gordonia planchonii H. Keng.), "ayahuasca" (Banisteriopsis caapi (Spruce), "chiric zanango" (Brunfelsia grandiflora D. Don), "pichirina" (Vismia angusta Aublet), "sangre de grado" (Croton lechleri Muell. Arg.), "ojé” (Ficus insipida Wild), "copaíba", "capinurí" (Maquira coriaceae (Korstem) C. C. Berg), y "uña de gato" (Uncaria guianensis (AUBL) Gmel). De acuerdo con muchos autores especialistas en el tema, los indígenas amazónicos utilizan masivamente estas especies con fines medicinales. Los colonos, aprovechando los conocimientos de los nativos, también aprovechan las plantas silvestres con estos fines. Entre las especies más utilizadas destacan "sangre de grado", "ubos", "huamanzamana", "catahua" (Hura crepitans), "pichirina" y "ojé". Es conveniente realizar estudios a nivel básico de estas especies medicinales, a fin de determinar los componentes activos y efectos secundarios para optimizar el uso con fines curativos.

Se consigna 43 especies (Anexo 1; ver el Cuadro 9 en SIHUAYO, www.siamazonia.org.pe), como alternativa para la industria de productos diferentes a la madera, sean éstos la corteza, productos extractivos, frutos, semilla, fibras y hojas. Existen grandes expectativas sobre el potencial de estas especies, pero muchas de ellas son comercializadas a otros países de forma ilegal, como materia prima para otros productos derivados, desconociendo el derecho intelectual de los pueblos indígenas.

En lo referente a las especies de importancia científica y ecológica, se identifica 39 especies consideradas de mayor valor ecológico (Anexo 1; ver el Cuadro 10 en SIHUAYO, www.siamazonia.org.pe). Éstas representan la vegetación con mayor cantidad de árboles por especie, lo que probablemente se debe a una mayor capacidad de adaptación de estas especies en ese hábitat y con mayor capacidad regenerativa, en comparación a las especies raras o de distribución restringida que en algún momento tuvieron una presencia importante en el área, por lo que necesitan mucha atención para impedir su extinción local (Anexo 1; ver el Cuadro 11 en SIHUAYO, www.siamazonia.org.pe).

\section{CONCLUSIONES Y RECOMENDACIONES}

1. Se identificó de forma prelimar 256 especies vegetales de importancia económica y ecológica dentro del JBAH.

2. Los usos locales de las especies vegetales, en el presente estudio, responden a la recopilación de información de material bibliográfico existente y a afirmaciones de las comunidades existentes dentro del área de influencia del JBAH. 
3. Destacan las especies por su uso como madera redonda para la construcción de viviendas rústicas, madera aserrada, en la medicina tradicional local y de uso alimentario.

4. Los usos locales son válidos para los caseríos de Puerto Almendra, Nina Rumi, Llanchama y otras poblaciones cercanas de la cuenca baja del río Nanay.

5. El manejo de información existente en el presente trabajo y su aplicación práctica responden a ciertas técnicas de orientación, destinadas a mejorar la calidad de un producto a base de madera y productos diferentes a la madera.

6. Se recomienda realizar trabajos de reforestación, con fines de enriquecimiento en áreas intervenidas, utilizando especies promisorias de rápido crecimiento y múltiples usos.

7. Se debe propiciar la realización de estudios detallados de las especies de uso medicinal, propuestas en el estudio para viabilizar su mejor aplicación.

8. Se debe realizar estudios tecnológicos aplicados de la madera aserrada de especies forestales a fin de determinar los usos definitivos.

9. Se recomienda realizar estudios de comercialización a nivel local de los productos de las especies vegetales estudiadas.

10. Se debe propiciar una campaña divulgativa de concienciación hacia las comunidades de la cuenca del río Nanay y afines, con la finalidad de enseñarles la conservación, manejo y uso de las especies identificadas como económica y ecológicamente importantes en el JBAH.

\section{AGRADECIMIENTOS}

Nuestro agradecimiento al proyecto BIODAMAZ, Perú - Finlandia, por la oportunidad de realizar este trabajo.

\section{BIBLIOGRAFÍA}

ARÓSTEGUI, A. 1974. Estudio Tecnológico de madera del Perú. Vol. I. Características Tecnológicas y Usos de las maderas de 145 especies del país. Dirección General de Investigación Agraria. UNALM. Lima, Perú. 483p.

ARÓSTEGUI, A. 1975. Características Tecnológicas y usos de la madera de 40 especies del bosque Nacional Alexander Von Humboldt. Ministerio de Agricultura. Dirección General de Investigación. Lima, Perú. $171 \mathrm{p}$.

ARÓSTEGUI, A. 1979. Estructura Integral de la Madera para construcción. Ministerio de Agricultura y Alimentación. UNALM, Dpto. de Industrias Forestales. Lima, Perú. 166p.

ARÓSTEGUI, A. 1982. Recopilación y Análisis de Estudio Tecnológicos de Madera Peruanas. Ministerio de Agricultura. PNUD. Lima, Perú. 57p.

ARÓSTEGUI, A.; SATO, A. 1970. Propiedades Físico Mecánicas de la Madera de 16 Especies Forestales del Perú. Revista Forestal del Perú 4 (1-2):13-24.

ARÓSTEGUI, A.; SOBRAL, M. 1986. Avance Tecnológico del Proyecto Usos de la Madera del Bosque Húmedo Tropical Colonia Angamos -Rio Yavarí y Jenaro Herrera. Convenio INPA-IIAP-UNAP. Iquitos, Perú. $14 \mathrm{p}$. 
ARÓSTEGUI, A.; VALDERRAMA, H. 1986. Usos de las maderas del bosque Húmedo tropical Allpahuayo Iquitos. UNAP - Facultad de Ingeniería Forestal. Convenio UNAP-IIAP. Iquitos, Perú. 32 p.

BEGAZO, N.; ARÓSTEGUI, A. 1985. Estructura Anatómica y clave de 20 especies forestales de Iquitos-Perú. Revista Forestal del Perú 1 (1): 3-22.

BIODAMAZ. 2004. Plan de desarrollo del Jardín Botánico-Arboretum El Huayo. Documento Técnico No 09. Serie BIODAMAZ-IIAP. Iquitos, Perú.

BUENO, J. 1970. Aptitud Papelera de 21 Especies Forestales del Perú. Revista Forestal del Perú 4 (1-2): 32-40.

DOUROJEANNI, M.J. 1981. Lineamientos generales para el desarrollo rural del área de influencia de la carretera Iquitos-Nauta. En: DGFF/ORDELOR. Evaluación y Lineamientos de Manejo de Suelos y Bosques para el Desarrollo Agrario del Area de Influencia de la Carretera Iquitos-Nauta. Loreto, Perú. pp. 283321.

ENCARNACIÓN, F. 1983. Nomenclatura de las Especies Forestales. Comunes en el Perú. Documento de Trabajo No. 7. Proyecto PNUD/FAO/PER/-81/002. Lima, Perú. 149 p.

ITTO - Asociación Internacional de Maderas Tropicales. 1991. Utilización Industrial de Nuevas Especies Forestales en el Perú. Fase I. Cámara Forestal Nacional. Dirección General Forestal y de Fauna. Proyecto ITTO PD/37/88. Lima, Perú. 46p.

JUNAC - Junta del Acuerdo de Cartagena. 1981a. Descripción General y Anatómica de 105 Maderas del Grupo Andino. PADT-REFORT/JUNAC. Grupo Andino. Cali, Colombia. 442p.

JUNAC - Junta del Acuerdo de Cartagena. 1981b. Tablas de Propiedades Físicas y Mecánicas de la Madera de 20 Especies del Perú. PADT-REFORT/JUNAC. Grupo Andino. Lima, Perú. 53p.

LAO, R.; FLORES, S. 1972. Arboles del Perú. Descripción de Algunas Especies Forestales de Jenaro Herrera, Iquitos. UNALM /COTESU. Iquitos, Perú. 195 p.

SIHUAYO - Sistema de Información del Jardín Botánico-Arboretum El Huayo. www.siamazonia.org.pe.

SOTO, S.T.; VÁSQUEZ, M.R. 1989. Maderas Redondas de Uso Estructural: Un material de Construcción a revalorar en la Selva Peruana. Asociación de Egresados Forestales (AEFAP). Consejo Nacional de Ciencia y Tecnología (CONCYTEC). Iquitos, Perú. 60p.

VALDERRAMA, H. 1984. Estudios de las Propiedades Físicas y su variación en el tronco de la Tachigalia longiflora Ducke y Sclerolobium melinonii Hans, en Puerto Almendra, Iquitos. Tesis para optar el título de Ingeniero Forestal, UNAP. Iquitos, Perú. 120p.

VALDERRAMA, H. 1992. Influencia de la estructura anatómica en el comportamiento tecnológico de 30 especies forestales de la Amazonía Peruana. Revista Conocimiento (UNAP) 2 (3): 13-23.

VALDERRAMA, H. 1993. Características Tecnológicas y uso Industrial de Nuevas especies forestales de la Amazonía Peruana. Folia Amazónica 5 (2): 73-93.

VALDERRAMA, H. 1998. Aspectos Ecológicos y Fitosociológicos de las especies forestales de la parcela II del Arboretum Amazónico del CIEFOR, Puerto Almendras, Iquitos. Revista Conocimiento (UNAP) 4 (1):36-74. 
VALDERRAMA, H. 2000a. Aspectos Fitosociológicos y Ecológicos de las especies forestales de la Parcela I del Arboretum Amazónico del CIEFOR, Puerto Almendras, Iquitos. Laboratorio de Anatomía y Tecnología de la Madera. FIF - UNAP. Boletín Técnico: Arboretum Amazónico. Serie: Fitosociología. Iquitos, Perú. 65p.

VALDERRAMA, H. 2000b. Aspectos Fitosociológicos y Ecológicos de las especies forestales de la Parcela III del Arboretum Amazónico del CIEFOR, Puerto Almendras, Iquitos. Laboratorio de Anatomía y Tecnología de la Madera. FIF - UNAP. Boletín Técnico: Arboretum Amazónico. Serie: Fitosociología. Iquitos, Perú. 65p.

VALDERRAMA, H. 2000c. Aspectos Fitosociológicos y Ecológicos de las especies forestales de la Parcela IV del Arboretum Amazónico del CIEFOR, Puerto Almendras, Iquitos. Laboratorio de Anatomía y Tecnología de la Madera. FIF - UNAP. Boletín Técnico: Arboretum Amazónico. Serie: Fitosociología. Iquitos, Perú.58p.

VALDERRAMA, H. 2000d. Aspectos Fitosociológicos y Ecológicos de las especies forestales de la Parcela V del Arboretum Amazónico del CIEFOR, Puerto Almendras. Iquitos. Laboratorio de Anatomía y Tecnología de la Madera. FIF - UNAP. Boletín Técnico: Arboretum Amazónico. Serie: Fitosociología. Iquitos, Perú. 68p.

VALDERRAMA, H.; TORRES, J. 1998. Cualidades Tecnológicas de uso de la madera de diez especies forestales de la Amazonía Peruana. Revista Conocimiento (UNAP) 4 (1): 17-35.

VALDERRAMA, H.; ARÓSTEGUI, A.; LOUREIRO, A. 1989. Estructura Anatómica y clave de identificación de 20 especies forestales de la zona de colonia Angamos - Río Yavarí y Jenaro Herrera. Convenio IIAP-UNAP. Iquitos, Perú. 140 p.

VARGAS, S. 1996. Diagnóstico Situacional de uso de Vegetales en Medicina Folklórica en la zona de Iquitos. Tesis para optar el título de Ingeniero Forestal. FIF - UNAP. Iquitos, Perú. 43 p.

VÁSQUEZ, R. 1989. Plantas Utiles de la Amazonía Peruana. I. Field Research Associate del Missouri Botanical Garden. Proyecto Flora del Perú. Apartado 280. Iquitos, Perú. 195 p. 
Anexo 1. Especies de importancia económica y ecológica en el Jardín Botánico-Arboretum El Huayo.

\begin{tabular}{|c|c|c|c|c|c|c|c|c|c|c|c|c|}
\hline \multirow[b]{3}{*}{ No } & \multirow[b]{3}{*}{ NOMBRE COMÚN } & \multirow[b]{3}{*}{ NOMBRE CIENTÍFICO } & \multirow[b]{3}{*}{ FAMILIA } & \multicolumn{7}{|c|}{ ESPECIES DE IMPORTANCIA ECONÓMICA Y CIENTÍFICA } & \multirow{3}{*}{$\begin{array}{c}\text { ESPECIES } \\
\text { RARAS O DE } \\
\text { IMPORTANCIA }\end{array}$} & \multirow{3}{*}{$\begin{array}{c}\text { ESPECIES } \\
\text { RARAS O DE } \\
\text { DISTRIBUCIÓN } \\
\text { RESTRINGIDA }\end{array}$} \\
\hline & & & & \multicolumn{7}{|c|}{ USOS LOCALES } & & \\
\hline & & & & $\begin{array}{r}\text { MADERA } \\
\text { REDONDA }\end{array}$ & \begin{tabular}{|c|} 
MADERA \\
ASERRADA \\
\end{tabular} & $\begin{array}{c}\text { USO } \\
\text { ALIMENTARIO } \\
\end{array}$ & $\begin{array}{c}\text { USO } \\
\text { ORNAMENTAL } \\
\end{array}$ & \begin{tabular}{|l} 
ARTESANÍA \\
INDUSTRIAL
\end{tabular} & MEDICINAL & USO & & \\
\hline 1 & Ipururo & Alchornea castaneifolia Will & EUPHORBIACEAE & & & & & & $\mathrm{x}$ (hojas y corteza) & $\mathrm{x}($ corteza $)$ & & \\
\hline 2 & Ipururo & Alchornea discolor E.P. & EUPHORBIACEAE & & & $\mathrm{x}$ (peces) & & & & & & $\mathrm{x}$ \\
\hline 3 & Zancudo caspi & Alchornea triplinervia (Benth) M. Arg & EUPHORBIACEAE & & & & & & & $\mathrm{x}($ corteza $)$ & $\mathrm{x}$ & \\
\hline 4 & Zancudo caspi & Alchorneopsis floribunda (B.) M.A. & EUPHORBIACEAE & & & & & & & & $\mathrm{x}$ & \\
\hline 5 & Shamoja & Amaioua guianensis H.B.K. & RUBIACEAE & & & & & $\mathrm{x}$ (madera) & $\mathrm{x}$ (corteza) & & & \\
\hline 6 & Sacha casho & Anacardium giganteum Hancock & ANACARDIACEAE & & $\mathrm{x}$ & $\mathrm{x}$ (personas $\mathrm{y}$ fauna) & & & & & & $\mathrm{x}$ \\
\hline 7 & Añuje moena & Anaueria brasiliensis Kosterm & LAURACEAE & $\mathrm{x}$ & $\mathrm{x}$ & $\mathrm{x}$ (personas $\mathrm{y}$ fauna) & & & & & $\mathrm{x}$ & \\
\hline 8 & Moena amarilla & Aniba amazonica (Meiz) Mez & LAURACEAE & $\mathrm{x}$ & $\mathrm{x}$ & & & & & & & \\
\hline 9 & Moena & Aniba hostmanniana (Nees) Mez & LAURACEAE & $\mathrm{x}$ & $\mathrm{x}$ & & & & & & & \\
\hline 10 & Moena & Aniba megaphylla $\mathrm{Mez}$ & LAURACEAE & $\mathrm{x}$ & $\mathrm{x}$ & & & & & & & \\
\hline 11 & Moena, moena amarilla & Aniba parviflora (Meissner) Mez & LAURACEAE & $\mathrm{x}$ & $\mathrm{x}$ & & & & & & & \\
\hline 12 & Palo de rosa & Aniba rosaeodora Ducke & LAURACEAE & $\mathrm{x}$ & $\mathrm{x}$ & & & & $\mathrm{x}$ (aceite, madera) & $\mathrm{x}$ (aceite) & & $\mathrm{x}$ \\
\hline 13 & Espintana de hoja menuda & Annona sp. & ANNONACEAE & $\mathrm{x}$ & & & & & & & & \\
\hline 14 & Pumaquiro & Aspidosperma macrocarpon Mart & APOCYNACEAE & $\mathrm{x}$ & $\mathrm{x}$ & & & & & & & \\
\hline 15 & Remo caspi & Aspidosperma rigidum Rusby & APOCYNACEAE & $\mathrm{x}$ & & & & $\mathrm{x}$ (tallo) & & $\mathrm{x}($ corteza $)$ & & \\
\hline 16 & Quillobordón negro & Aspidosperma spruceanum B. ex M.A. & APOCYNACEAE & & $\mathrm{x}$ & & & & & & & \\
\hline 17 & Quillobordon & Aspidosperma vargasii A.D.C. & APOCYNACEAE & & $\mathrm{x}$ & & & & & & & \\
\hline 18 & Chambira & Astrocaryum chambira Burret & ARECACEAE & & & $\mathrm{x}$ (personas $\mathrm{y}$ fauna) & & $\mathrm{x}($ cogollo $)$ & & & $\mathrm{x}$ & \\
\hline 19 & Inayuga & Attalea maripa (Aublet) Mart & ARECACEAE & & & & $\mathrm{x}$ & & & & & \\
\hline 21 & Ayahuasca & Banisteriopsis caapi (Spruce) & MALPIGHIACEAE & & & & & & $\mathrm{x}$ (liana, extractos) & $\mathrm{x}$ (capsulas) & & \\
\hline 20 & Huairuro colorado & Batesia floribunda Spruce & FABACEAE & & $\mathrm{x}$ & & & $\mathrm{x}$ (semilla) & $\mathrm{x}($ corteza $)$ & & & \\
\hline 22 & Castaña & Bertholletia excelsa H.\&.B. & LECYTHIDACEAE & & $\mathrm{x}$ & $\mathrm{x}$ (personas $\mathrm{y}$ fauna $)$ & & & & $\mathrm{x}$ (aceite) & $\mathrm{x}$ & \\
\hline 23 & Chingonga & Brosimum parinarioides Ducke & MORACEAE & & & & & & & & $\mathrm{x}$ & \\
\hline 24 & Palisangre & Brosimum rubescens Taubert & MORACEAE & & & & & $\mathrm{x}$ (madera) & $\mathrm{x}$ (madera) & & $\mathrm{x}$ & \\
\hline 25 & Chingonga & Brosimum utile (H.B.K.) Pittier & MORACEAE & & $\mathrm{x}$ & & & & $\mathrm{x}($ latex) & $\mathrm{x}($ latex) & $\mathrm{x}$ & \\
\hline 26 & Chiric sanango & Brunfelsia grandiflora D. Don & SOLANACEAE & & & & $\mathrm{x}$ & & x(raíz) & x(raíz) & & \\
\hline 27 & Indano & Byrsonima coriaceae $(\mathrm{SW}) \mathrm{DC}$ & MALPIGHIACEAE & & & & & & $\mathrm{x}$ (corteza) & & & \\
\hline 28 & Lagarto caspi & Calophyllum brasiliense Cambers & CLUSIACEAE & $\mathrm{x}$ & $\mathrm{x}$ & & & & & & & \\
\hline 29 & Huacapurana & Campsiandra angustifolia Spring & FABACEAE & & & & & & $\mathrm{x}$ (corteza y madera) & & & \\
\hline 30 & Brea caspi & Caraipa densifolia subsp. densifolia Mart & CLUSIACEAE & $\mathrm{x}$ & $\mathrm{x}$ & & & & & & & \\
\hline 31 & Aceite caspi blanco & Caraipa tereticaulis Tulasne & CLUSIACEAE & $\mathrm{x}$ & & & & & & & & \\
\hline 32 & Aceite caspi negro & Caraipa utiles Vásquez & CLUSIACEAE & & & & & & & & & \\
\hline 33 & Andiroba & Carapa guianensis Aubl & MELIACEAE & & $\mathrm{x}$ & & & & & & & \\
\hline 34 & Cachimbo caspi, tahuarí & Cariniana decandra Ducke & LECYTHIDACEAE & & $\mathrm{x}$ & & & $\mathrm{x}$ (fruto) & $\mathrm{x}$ (corteza) & & $\mathrm{x}$ & \\
\hline 35 & Papelillo caspi & Cariniana multiflora Ducke & LECYTHIDACEAE & & $\mathrm{x}$ & & & & & & & \\
\hline 36 & Almendro & Caryocar glabrum (Aublet) Persoon & CARYOCARACEAE & & $\mathrm{x}$ & $\mathrm{x}$ (personas $\mathrm{y}$ fauna) & & & & & $\mathrm{x}$ & \\
\hline 37 & Almendro & Caryocar microcarpum Ducke & CARYOCARACEAE & & $\mathrm{x}$ & $\mathrm{x}$ (personas $\mathrm{y}$ fauna) & & & & & & \\
\hline 38 & Cedro & Cedrela odorata $\mathrm{L}$. & MELIACEAE & & $\mathrm{x}$ & & $\mathrm{x}$ & $\mathrm{x}$ (frutos) & $\mathrm{x}$ (corteza) & & & \\
\hline 39 & Tornillo, huayra caspi & Cedreling a cateniformis Ducke & MIMOSACEAE & & $\mathrm{x}$ & & & & $\mathrm{x}$ (corteza) & & $\mathrm{x}$ & \\
\hline 40 & Lupuna & Ceiba pentandra $(\mathrm{L})$ Faertn & BOMBACACEAE & & $\mathrm{x}$ & & & $\mathrm{x}$ (algodon) & & & & \\
\hline 41 & Huimba & Ceiba samauma Aublet & BOMBACACEAE & & $\mathrm{x}$ & & & $x$ (fruto) & & & & \\
\hline$\underline{42}$ & Quillosisa & Cespedecia spathulata (R.P.) Planch & OCHNACEAE & & $\mathrm{x}$ & & & & & & & \\
\hline
\end{tabular}




\begin{tabular}{|c|c|c|c|c|c|c|c|c|c|c|c|c|}
\hline \multirow[b]{3}{*}{ No } & \multirow[b]{3}{*}{ NOMBRE COMÚN } & \multirow[b]{3}{*}{ NOMBRE CIENTÍFICO } & \multirow[b]{3}{*}{ FAMILIA } & \multicolumn{7}{|c|}{ ESPECIES DE IMPORTANCIA ECONÓMICA Y CIENTÍFICA } & \multirow{3}{*}{$\begin{array}{c}\text { ESPECIES } \\
\text { RARAS O DE } \\
\text { IMPORTANCIA }\end{array}$} & \multirow{3}{*}{\begin{tabular}{|c|} 
ESPECIES \\
RARAS O DE \\
DISTRIBUCIÓN \\
RESTRINGIDA \\
\end{tabular}} \\
\hline & & & & \multicolumn{7}{|c|}{ USOS LOCALES } & & \\
\hline & & & & \begin{tabular}{|c|} 
MADERA \\
REDONDA \\
\end{tabular} & \begin{tabular}{|c|} 
MADERA \\
ASERRADA \\
\end{tabular} & $\begin{array}{c}\text { USO } \\
\text { ALIMENTARIO }\end{array}$ & $\begin{array}{c}\text { USO } \\
\text { ORNAMENTAL }\end{array}$ & $\begin{array}{l}\text { ARTESANÍA } \\
\text { INDUSTRIAL } \\
\end{array}$ & MEDICINAL & USO & & \\
\hline 43 & Mashonaste, tulplay & Clarisia racemosa $\mathrm{R} \& \mathrm{~F}$ & MORACEAE & & $\mathrm{x}$ & & & $\mathrm{x}(\mathrm{rama})$ & $\mathrm{x}($ latex) & & $\mathrm{x}$ & \\
\hline 44 & Cumala & Compsoneura capitellata (A.DC.) Warb. & MYRISTICACEAE & $\mathrm{x}$ & $\mathrm{x}$ & & & & & & & \\
\hline 45 & Copaíba & Copaifera paupera (Hars) S. & FABACEAE & & $\mathrm{x}$ & & & & $\mathrm{x}$ (aceite, madera) & $x$ (aceite) & & $\mathrm{x}$ \\
\hline 46 & Añallu caspi & Cordia ucayaliensis I.M. Johnston & BORAGINACEAE & $\mathrm{x}$ & & & & & & & & \\
\hline 47 & Acero caspi & Cosmibuena grandiflora $(\mathrm{R} \& \mathrm{P})$ & RUBIACEAE & $\mathrm{x}$ & & & & & & & & \\
\hline 48 & Leche caspi & Couma macrocarpa Barbosa Rodrigues & APOCYNACEAE & & & $\mathrm{x}$ (personas $\mathrm{y}$ fauna) & & & $\mathrm{x}($ latex) & $\mathrm{x}($ latex) & $\mathrm{x}$ & \\
\hline 49 & Copal blanco & Crepidospermum prancei Daly & BURSERACEAE & $\mathrm{x}$ & $\mathrm{x}$ & & & & & & $\mathrm{x}$ & \\
\hline 50 & Copal & Crepidospermum rhoifolium (Benth)S.w. & BURSERACEAE & $\mathrm{x}$ & $\mathrm{x}$ & & & & & & & \\
\hline 51 & Sangre de grado & Croton lechleri Muell. Arg & EUPHORBIACEAE & & & & & & $\mathrm{x}($ latex) & $\mathrm{x}($ latex) & & \\
\hline 52 & Quinilla & Chrysophyllum bombycinum Pennington & SAPOTACEAE & $\mathrm{x}$ & & $\mathrm{x}$ (personas $\mathrm{y}$ fauna) & & $\mathrm{x}$ (madera) & & $\mathrm{x}($ latex) & & \\
\hline 53 & Quinilla colorada & Chrysophyllum manaosense (A.) P. & SAPOTACEAE & $\mathrm{x}$ & & $\mathrm{x}$ (personas $\mathrm{y}$ fauna) & & $\mathrm{x}$ (madera) & & $\mathrm{x}($ latex $)$ & $\mathrm{x}$ & \\
\hline 54 & Garza moena & Dendropanax umbellatum (R.P.) Donn & ARALIACEAE & & $\mathrm{x}$ & & & & & & & \\
\hline 55 & Azucar huaillo & Dialium guianensis (Aublet) Sandwith & FABACEAE & $\mathrm{x}$ & & $\mathrm{x}$ (personas $\mathrm{y}$ fauna) & & & $\mathrm{x}($ corteza $)$ & & & \\
\hline 56 & Moena & Diplotropis pupurea (Richard) Amsh. & FABACEAE & $\mathrm{x}$ & & & & & & & & \\
\hline 57 & Charapilla & Dipteryx odorata (Aublet) Willd. & FABACEAE & & & $\mathrm{x}$ (personas $\mathrm{y}$ fauna) & & $\mathrm{x}$ (semilla) & & $\mathrm{x}($ aceite $)$ & & $\mathrm{x}$ \\
\hline 58 & Paujil chaqui (liana) & Doliocarpus dentatus (Aubl) Stand & DILLENACEAE & & & & & $\mathrm{x}($ tallo $) \mathrm{x}($ & gua de la parte medu & tar) & & \\
\hline 59 & Yutubanco & Drypetes amazonica var peruviana Macbr & EUPHORBIACEAE & $\mathrm{x}$ & & & & & & & & \\
\hline 60 & Carahuasca negra & Duguetia tessmannii R.E. Fries & ANNONACEAE & $\mathrm{x}$ & $\mathrm{x}$ & & & & & & & \\
\hline 61 & Pampa remo caspi & Duroia paraensis Ducke & RUBIACEAE & $\mathrm{x}$ & & & & & & & & \\
\hline 62 & Quinilla & Ecclinusa lanceolata (C.M.\&.E.) Pierre & SAPOTACEAE & $\mathrm{x}$ & & $\mathrm{x}$ (personas $\mathrm{y}$ fauna) & & $\mathrm{x}$ (madera) & & & & $\mathrm{x}$ \\
\hline 63 & Machimango & Eschweilera coriacea (A.DC.) S. Mori. & LECYTHIDACEAE & $\mathrm{x}$ & & & & & & & $\mathrm{x}$ & \\
\hline 64 & Machimango & Eschweilera grandiflora (Aubl) Sandw & LECYTHIDACEAE & $\mathrm{x}$ & & & & & & & $\mathrm{x}$ & \\
\hline 65 & Machimango & Eschweilera itayensis Knuth & LECYTHIDACEAE & $\mathrm{x}$ & & & & & & & $\mathrm{x}$ & \\
\hline 66 & Machimango & Eschweilera tessmannii Knuth & LECYTHIDACEAE & $\mathrm{x}$ & & & & & & & $\mathrm{x}$ & \\
\hline 67 & Machimango & Eschweilera turbinata (Berg.) $\mathrm{Ndz}$ & LECYTHIDACEAE & $\mathrm{x}$ & & & & & & & & \\
\hline 68 & Huasai & Euterpe precatoria C. Martius & ARECACEAE & $\mathrm{x}$ & & $\mathrm{x}$ (personas $\mathrm{y}$ fauna) & $\mathrm{x}$ & x(estípite) & x(raíces) & $\mathrm{x}$ (chonta) & $\mathrm{x}$ & \\
\hline 69 & Renaquillo & Ficus americana Standl & MORACEAE & $\mathrm{x}$ & & & & & $\mathrm{x}$ (latex del tallo) & & & \\
\hline 70 & Ojé & Ficus insipida Wild & MORACEAE & & $\mathrm{x}$ & & & & $\mathrm{x}($ latex $)$ & $\mathrm{x}$ (latex) & & \\
\hline 71 & Plumero caspi & Froesia occidentalis Gerean y Vasquez & QUINIACEAE & & & & & & & & & $\mathrm{x}$ \\
\hline 72 & Charichuelo & Garcinia macrophylla C. Martius & CLUSIACEAE & & & $\mathrm{x}$ (personas $\mathrm{y}$ fauna) & $\mathrm{x}$ & & & & & \\
\hline 73 & Huito & Genipa americana Will & RUBIACEAE & & $\mathrm{x}$ & $\mathrm{x}$ (personas $\mathrm{y}$ fauna) & $\mathrm{x}$ & $\mathrm{x}$ (madera) & $\mathrm{x}$ (fruto) & $x$ (fruto verde) & & \\
\hline 74 & Ipururo de altura & Gordonia planchonii H. Keng. & THEACEAE & $\mathrm{x}$ & $\mathrm{x}$ & & & & $\mathrm{x}$ (hojas y corteza) & & & \\
\hline 75 & Requia & Guarea glabra M. Vahl & MELIACEAE & $\mathrm{x}$ & $\mathrm{x}$ & & & & & & & \\
\hline 76 & Requia & Guarea grandifolia DC. & MELIACEAE & $\mathrm{x}$ & $\mathrm{x}$ & & & & & & & \\
\hline 77 & Requia & Guarea juglandiformis Pennington & MELIACEAE & $\mathrm{x}$ & $\mathrm{x}$ & & & & $\mathrm{x}$ (corteza y hojas) & & & $\mathrm{x}$ \\
\hline 78 & Requia & Guarea kunthiana Adr. Jessieu & MELIACEAE & & & & & & & & & $\mathrm{x}$ \\
\hline 79 & Requia & Guarea macrophylla M. Vahl & MELIACEAE & $\mathrm{x}$ & $\mathrm{x}$ & & & & & & & \\
\hline 80 & Carahuasca & Guatteria elata R. E. Fries & ANNONACEAE & $\mathrm{x}$ & $\mathrm{x}$ & & $\mathrm{x}$ & & & & & \\
\hline 81 & Carahuasca & Guatteria hyposericea Diels & ANNONACEAE & $\mathrm{x}$ & $\mathrm{x}$ & & & & & & & \\
\hline 82 & Vara, carahuasca & Guatteria megalophylla Diels & ANNONACEAE & $\mathrm{x}$ & & & & & & & & \\
\hline 83 & Vara, carahuasca & Guatteria melosma Diels & ANNONACEAE & $\mathrm{x}$ & & & & & & & & \\
\hline 84 & Vara, carahuasca & Guatteria multinervia Diels & ANNONACEAE & $\mathrm{x}$ & & & & & & & & \\
\hline 85 & Carahuasca & Guatteria schomburgkiana C. Martius & ANNONACEAE & $\mathrm{x}$ & $\mathrm{x}$ & & & & & & $\mathrm{x}$ & \\
\hline
\end{tabular}




\begin{tabular}{|c|c|c|c|c|c|c|c|c|c|c|c|c|}
\hline \multirow[b]{3}{*}{ No } & \multirow[b]{3}{*}{ NOMBRE COMÚN } & \multirow[b]{3}{*}{ NOMBRE CIENTÍFICO } & \multirow[b]{3}{*}{ FAMILIA } & \multicolumn{7}{|c|}{ ESPECIES DE IMPORTANCIA ECONÓMICA Y CIENTÍFICA } & \multirow{3}{*}{$\begin{array}{c}\text { ESPECIES } \\
\text { RARAS O DE } \\
\text { IMPORTANCIA }\end{array}$} & \multirow{3}{*}{\begin{tabular}{|c} 
ESPECIES \\
RARAS O DE \\
DISTRIBUCIÓN \\
RESTRINGIDA \\
\end{tabular}} \\
\hline & & & & \multicolumn{7}{|c|}{ USOS LOCALES } & & \\
\hline & & & & \begin{tabular}{|c|} 
MADERA \\
REDONDA \\
\end{tabular} & \begin{tabular}{|c|} 
MADERA \\
ASERRADA \\
\end{tabular} & $\begin{array}{c}\text { USO } \\
\text { ALIMENTARIO }\end{array}$ & \begin{tabular}{|c|} 
USO \\
ORNAMENTAL
\end{tabular} & \begin{tabular}{|l|} 
ARTESANÍA \\
INDUSTRIAL \\
\end{tabular} & MEDICINAL & USO & & \\
\hline 86 & Carahuasca negra & Guatteria tomentosa (Rusby) & ANNONACEAE & $\mathrm{x}$ & $\mathrm{x}$ & & & & & & & $\mathrm{x}$ \\
\hline 87 & Misho chaqui & Helicostylis scabra (J.F.M.)C.C.B. & MORACEAE & & $\mathrm{x}$ & $\mathrm{x}$ (personas $\mathrm{y}$ fauna) & & & $\mathrm{x}($ latex $)$ & & & \\
\hline 88 & Tamshy (liana) & Heteropsis oblongifolia Kunth & ARACEAE & & & & & $\mathrm{x}$ (liana) & & & & \\
\hline 89 & Azucar huayo & Himenaea oblonguifolia var palustris & FABACEAE & & $\mathrm{x}$ & $\mathrm{x}$ (personas $\mathrm{y}$ fauna) & & & $x($ corteza $)$ & & & \\
\hline 90 & Azucar huayo & Hymenaea courbaril var. courbaril & FABACEAE & & $\mathrm{x}$ & $\mathrm{x}$ (fauna) & & & $x($ corteza $)$ & & & \\
\hline 91 & Azucar huayo & Hymenaea oblongifolia Huber var. oblongi & gifolia FABACEAE & & $\mathrm{x}$ & $\mathrm{x}$ (fauna) & & & $\mathrm{x}($ corteza $)$ & & & \\
\hline 92 & Mari mari negro & Hymenolobium excelsum Ducke & FABACEAE & & $\mathrm{x}$ & & & & & & $\mathrm{x}$ & \\
\hline 93 & Mari mari & Hymenolobium pulcherrimum Ducke & FABACEAE & & $\mathrm{x}$ & & & & & & & \\
\hline 94 & Shimbillo & Inga aria J. F. Macb. & FABACEAE & & & $\mathrm{x}$ (personas $\mathrm{y}$ fauna) & & & & & & $\mathrm{x}$ \\
\hline 95 & Shimbillo & Inga brachyrhachis Harms & FABACEAE & & & $\mathrm{x}$ (personas $\mathrm{y}$ fauna) & & & & & & \\
\hline 96 & Shimbillo & Inga ruiziana $\mathrm{G}$. Don & FABACEAE & & & $\mathrm{x}$ (personas $\mathrm{y}$ fauna) & & & & & & \\
\hline 97 & Shimbillo & Inga thibaudiana DC. & FABACEAE & & & $\mathrm{x}$ (personas $\mathrm{y}$ fauna) & & & & & & \\
\hline 98 & Cumala colorada & Iryanthera crassifolia A.C. Smith & MYRISTICACEAE & $\mathrm{x}$ & $\mathrm{x}$ & & & & & & & \\
\hline & Cumala colorada & Iryanthera elliptica Ducke & MYRISTICACEAE & $\mathrm{x}$ & $\mathrm{x}$ & & & & & & & \\
\hline & Cumala & Iryanthera laevis Markgraf & MYRISTICACEAE & & & & & & & & & $\mathrm{x}$ \\
\hline & Cumala colorada & Iryanthera lancifolia Ducke & MYRISTICACEAE & $\mathrm{x}$ & & & & & & & & \\
\hline & Cumala colorada & Iryanthera macrophylla (Benth) Warb. & MYRISTICACEAE & $\mathrm{x}$ & $\mathrm{x}$ & & & & & & & \\
\hline 103 & Cumala colorada & Iryanthera paraensis Huber & MYRISTICACEAE & & $\mathrm{x}$ & & & & & & $\mathrm{x}$ & \\
\hline 104 & Pucuna caspi & Iryanthera tricornis Ducke & MYRISTICACEAE & & $\mathrm{x}$ & & & $x$ (fruto $y$ tallo) & & & $\mathrm{x}$ & \\
\hline & Huamanzamana, ishtapi & Jacaranda copaia (Aubl.) D. Don & BIGNONIACEAE & $\mathrm{x}$ & & & & (semilla y madera) & $\mathrm{x}$ (hojas) & & $\mathrm{x}$ & \\
\hline 106 & Chicle huayo & Lacmellea arborescens (M. Arg)Mark. & APOCYNACEAE & & & & & & & & & \\
\hline 107 & Chicle huayo & Lacmellea floribunda (Poep.) & APOCYNACEAE & $\mathrm{x}$ & & $\mathrm{x}$ (personas $\mathrm{y}$ fauna) & $\mathrm{x}$ & & $\mathrm{x}$ (latex) & & & \\
\hline 108 & Chicle huayo & Lacmellea peruviana (Van H.\&.M.Arg.)M. & M. APOCYNACEAE & $\mathrm{x}$ & & $\mathrm{x}$ (personas $\mathrm{y}$ fauna) & & & & $\mathrm{x}$ (latex) & & \\
\hline & Palo de fundo & Ladenbergia magnifolia (R.\&.P.) Klot. & RUBIACEAE & & & & & & $\mathrm{x}($ corteza $)$ & & & \\
\hline 110 & Parinari & Licania bracteata Prance & CHRYSOBALANACEAE & & & $\mathrm{x}$ (personas $\mathrm{y}$ fauna) & & & & & & \\
\hline 111 & Parinari, chullachaqui & Licania heteromorpha Benth & CHRYSOBALANACEAE & $\mathrm{x}$ & & $\mathrm{x}$ (fauna) & & & & & & \\
\hline 112 & Parinari & Licania hypoleuca Bentham & CHRYSOBALANACEAE & $\mathrm{x}$ & & & & & & & & $\mathrm{x}$ \\
\hline 113 & Apacharama & Licania longistyla (Hoodrf.) Fristsch $\mathrm{Cl}$ & CHRYSOBALANACEAE & $\mathrm{x}$ & & & & $\mathrm{x}($ corteza $)$ & & & & \\
\hline 114 & Parinari & Licania macrocarpa Cuatrecasas & CHRYSOBALANACEAE & $\mathrm{x}$ & & $\mathrm{x}$ (personas $\mathrm{y}$ fauna) & & & & & & $\mathrm{x}$ \\
\hline 115 & Parinari & Licania octandra Prance & CHRYSOBALANACEAE & & & $\mathrm{x}$ (personas $\mathrm{y}$ fauna) & & & & & $\mathrm{x}$ & \\
\hline 116 & Parinari & Licania unguiculata Prance & CHRYSOBALANACEAE & & & $\mathrm{x}$ (personas $\mathrm{y}$ fauna) & & & & & & \\
\hline 117 & Moena & Licaria sp. & LAURACEAE & & & & & & & $\mathrm{x}($ aceite $)$ & & \\
\hline 118 & Sacha shimbillo & Macrolobium limbatum Spruce ex Benth. & FABACEAE & $\mathrm{x}$ & & $\mathrm{x}$ (personas $\mathrm{y}$ fauna) & & & & & & \\
\hline 119 & Capinurí & Maquira coriaceae (Korstem) C. C. Berg & MORACEAE & & $\mathrm{x}$ & & & $\mathrm{x}$ (rama) & $\mathrm{x}$ (latex) & & & \\
\hline 120 & Sacha bubinsana & Marmaroxylon basijugum (D) L.Rico & FABACEAE & & & & & & $\mathrm{x}($ corteza $)$ & & $\mathrm{x}$ & \\
\hline 121 & Caiman caspi & Matayba oligandra Sandw. & SAPINDACEAE & & & & & & & & & $\mathrm{x}$ \\
\hline 122 & Machin sapote & Matisia bracteolosa Ducke & BOMBACACEAE & & & $x$ (fauna) & & & & & & \\
\hline 123 & Machin sapote & Matisia malacocalyx (Rob. \& Nils.) & BOMBACACEAE & & & & & & & & & $\mathrm{x}$ \\
\hline 124 & Machin sapote & Matisia oblongifolia Poepp \& Endl & BOMBACACEAE & & & $\mathrm{x}$ (fauna) & & & & & & \\
\hline 125 & Machin sapote & Matisia ochrocalyx Schum. & BOMBACACEAE & & & $\mathrm{x}$ (fauna) & & & $\mathrm{x}$ (hojas) & & & \\
\hline 126 & Aguaje & Mauritia flexuosa L.F. & ARECACEAE & & & $\mathrm{x}$ (personas $\mathrm{y}$ fauna) & $\mathrm{x}$ & $x$ (fruto) & & $\mathrm{x}$ (crema) & & \\
\hline 127 & Chuchuhuasha & Maytenus macrocarpa $(\mathrm{R} \& \mathrm{P})$ Briq & CELASTRACEAE & & & & & & $\mathrm{x}($ corteza $)$ & & & \\
\hline 128 & Tahuari negro & Memora cladotricha Sandwith & BIGNONIACEAE & & & & & & & & & $\mathrm{x}$ \\
\hline
\end{tabular}




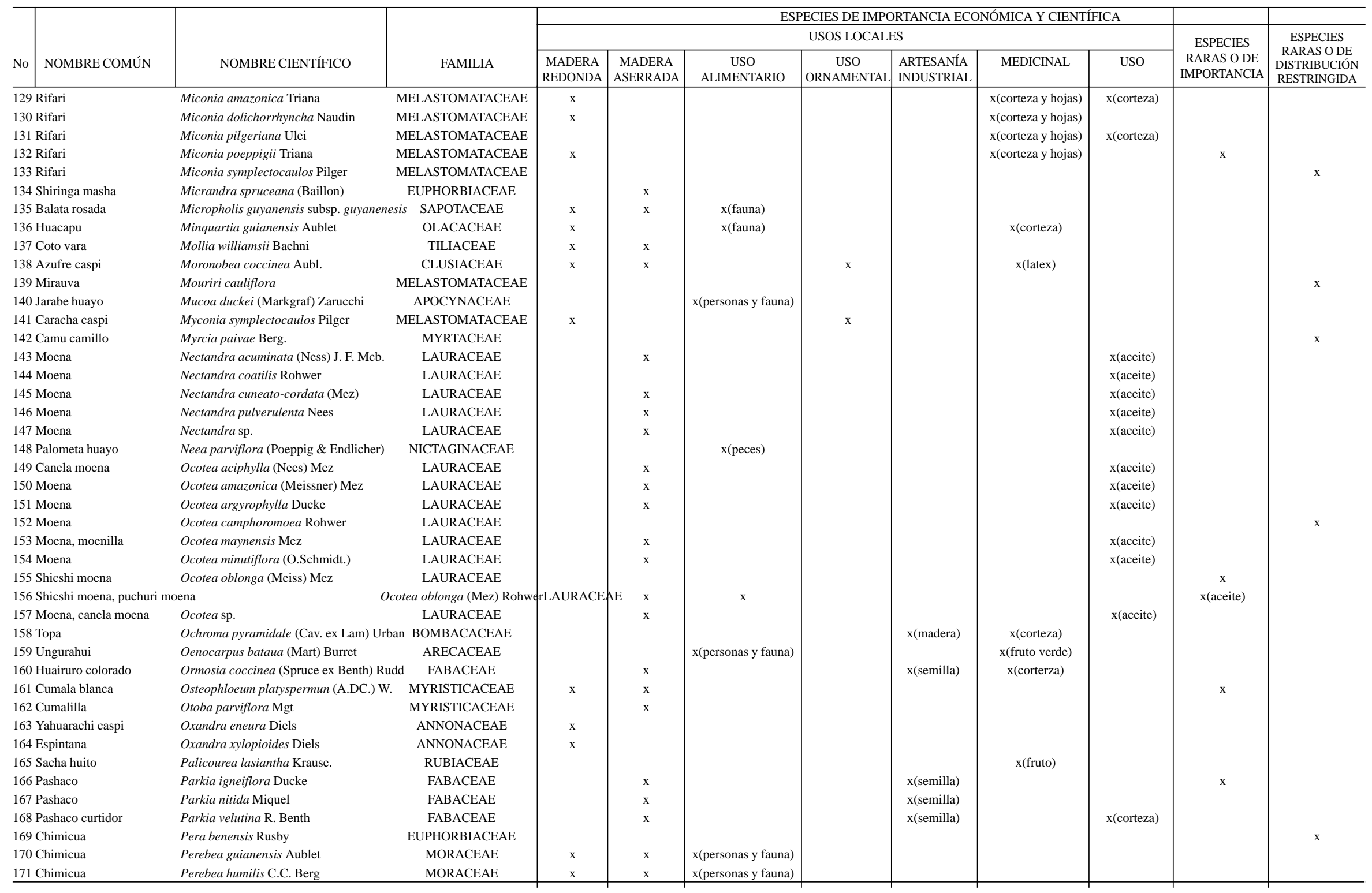




\begin{tabular}{|c|c|c|c|c|c|c|c|c|c|c|c|c|}
\hline \multirow[b]{3}{*}{ No } & \multirow[b]{3}{*}{ NOMBRE COMÚN } & \multirow[b]{3}{*}{ NOMBRE CIENTÍFICO } & \multirow[b]{3}{*}{ FAMILIA } & \multicolumn{7}{|c|}{ ESPECIES DE IMPORTANCIA ECONÓMICA Y CIENTÍFICA } & \multirow{3}{*}{$\begin{array}{c}\text { ESPECIES } \\
\text { RARAS O DE } \\
\text { IMPORTANCIA }\end{array}$} & \multirow{3}{*}{$\begin{array}{c}\text { ESPECIES } \\
\text { RARAS O DE } \\
\text { DISTRIBUCIÓN } \\
\text { RESTRINGIDA } \\
\end{array}$} \\
\hline & & & & \multicolumn{7}{|c|}{ USOS LOCALES } & & \\
\hline & & & & \begin{tabular}{|r|} 
MADERA \\
REDONDA \\
\end{tabular} & \begin{tabular}{|c|} 
MADERA \\
ASERRADA \\
\end{tabular} & $\begin{array}{c}\text { USO } \\
\text { ALIMENTARIO }\end{array}$ & \begin{tabular}{|c|} 
USO \\
ORNAMENTAL
\end{tabular} & $\begin{array}{l}\text { ARTESANÍA } \\
\text { INDUSTRIAL }\end{array}$ & MEDICINAL & USO & & \\
\hline \multicolumn{2}{|c|}{172 Chimicua } & Perebea longepedunculata & MORACEAE & & & & & & & & & $\mathrm{x}$ \\
\hline \multicolumn{2}{|c|}{173 Moena } & Pleurothyrium panurense (Meissner) Mez & LAURACEAE & & $\mathrm{x}$ & & & & & $\mathrm{x}$ (aceite) & & \\
\hline \multicolumn{2}{|c|}{174 Canela moena } & Pleurothyrium parviflorum Ducke & LAURACEAE & & $\mathrm{x}$ & & & & & $\mathrm{x}($ aceite $)$ & & \\
\hline \multicolumn{2}{|c|}{175 Moena } & Pleurothyrium sp. & LAURACEAE & & $\mathrm{x}$ & & & & & $\mathrm{x}$ (aceite) & & \\
\hline \multicolumn{2}{|c|}{176 Yanavara, ocuera negra } & Pollalesta discolor (HBK) Arist. & ASTERACEAE & $\mathrm{x}$ & & & & & & & & \\
\hline \multicolumn{2}{|c|}{177 Humari } & Poraqueiba sericea Tulasne & ICACINACEAE & & & $\mathrm{x}$ (personas $\mathrm{y}$ fauna) & & & & & & \\
\hline \multicolumn{2}{|c|}{178 Sacha uvilla } & Pourouma guianensis Aublet & CECROPIACEAE & & & $\mathrm{x}$ (fauna) & & & & & & \\
\hline \multicolumn{2}{|c|}{179 Sacha uvilla } & Pourouma mollis Trécul & CECROPIACEAE & & & $\mathrm{x}$ (personas $\mathrm{y}$ fauna) & & & & & & \\
\hline \multicolumn{2}{|c|}{180 Sacha uvilla } & Pourouma ovata Trécul & CECROPIACEAE & & & $\mathrm{x}$ (personas $\mathrm{y}$ fauna) & & & & & & \\
\hline \multicolumn{2}{|c|}{181 Sacha uvilla } & Pourouma tomentosa $\mathrm{C}$. Martius & CECROPIACEAE & & & $\mathrm{x}$ (fauna) & & & & & & \\
\hline \multirow{2}{*}{\multicolumn{2}{|c|}{$\begin{array}{l}182 \text { Quinilla, caimitillo } \\
183 \text { Quinilla }\end{array}$}} & Pouteria aff. caimito (R. et P) Randk & SAPOTACEAE & $\mathrm{x}$ & & $\mathrm{x}$ (personas $\mathrm{y}$ fauna) & & & & $\mathrm{x}($ latex) & $\mathrm{x}$ & $\mathrm{x}$ \\
\hline & & Pouteria guianensis Aublet & SAPOTACEAE & $\mathrm{x}$ & & $\mathrm{x}$ (personas $\mathrm{y}$ fauna) & & $\mathrm{x}$ (madera) & & & & \\
\hline \multicolumn{2}{|c|}{184 Quinilla } & Pouteria wurdackii Aubl & SAPOTACEAE & $\mathrm{x}$ & & $\mathrm{x}$ (personas $\mathrm{y}$ fauna) & & $\mathrm{x}($ madera $)$ & & & & \\
\hline & Copal blanco & Protium altsonii Sandwith & BURSERACEAE & $\mathrm{x}$ & $\mathrm{x}$ & $\mathrm{x}$ (personas $\mathrm{y}$ fauna) & & & & & & $\mathrm{x}$ \\
\hline & Copal & Protium ferrugineum (Engler) Engler & BURSERACEAE & $\mathrm{x}$ & $\mathrm{x}$ & & & & & & & \\
\hline & Copal & Protium gallosum Daly & BURSERACEAE & $\mathrm{x}$ & $\mathrm{x}$ & & & & & & & \\
\hline & Copal & Protium grandifolium Engler & BURSERACEAE & $\mathrm{x}$ & $\mathrm{x}$ & & & & & & & \\
\hline & Copal & Protium guianensis Daly & BURSERACEAE & $\mathrm{x}$ & $\mathrm{x}$ & & & & & & & \\
\hline & Copal & Protium hebetatum Vel sp. aff & BURSERACEAE & $\mathrm{x}$ & $\mathrm{x}$ & & & & & & & $\mathrm{x}$ \\
\hline & Copal & Protium nodulosum Swart & BURSERACEAE & $\mathrm{x}$ & $\mathrm{x}$ & & & & & & & $\mathrm{x}$ \\
\hline & Copal & Protium subserratum Engler & BURSERACEAE & $\mathrm{x}$ & $\mathrm{x}$ & & & & & & & \\
\hline & Copal & Protium trifoliolatum Engler & BURSERACEAE & $\mathrm{x}$ & $\mathrm{x}$ & & & & & & & $\mathrm{x}$ \\
\hline & Siririca, espintana & Pseudoxandra polypheba (Diels) R. E. Fries & $S$ ANNONACEAE & $\mathrm{x}$ & & & & & & & & \\
\hline & Chontaquiro & Pterocarpus rohrii M. Vahl & FABACEAE & $\mathrm{x}$ & $\mathrm{x}$ & & & & & & & \\
\hline & Quillosisa & Qualea paraensis Ducke & VOCHIZIACEAE & $\mathrm{x}$ & & & & & & & & \\
\hline & Cascarilla & Remijia peruviana Standley & RUBIACEAE & & & & $\mathrm{x}$ & & $\mathrm{x}$ (corteza) & & & \\
\hline & Mishqui panga & Renealmia alpinia (Roff) Para. & ZINGIBERACEAE & & & $\mathrm{x}$ (personas) & $\mathrm{x}$ & $\mathrm{x}($ tinte $)$ & $\mathrm{x}$ (hoja) & $\mathrm{x}$ (fruto) & & \\
\hline & Charichuelo de hoja menuda & Rheedia gardneriana Miers. Ex Plan. et Triana & a CLUSIACEAE & $\mathrm{x}$ & & $\mathrm{x}$ (personas $\mathrm{y}$ fauna) & & & & & & \\
\hline & Punguilla & Rhodognaphalopsis brevipes A. Robyns & BOMBACACEAE & $\mathrm{x}$ & & & & & & & & \\
\hline & Marupa negro & Simaba orinocensis H.B.K. & SIMAROUBACEAE & $\mathrm{x}$ & & & & & & & & \\
\hline & Marupa negro & Simaba polyphilla (Cavalac.) W. Thomas & SIMAROUBACEAE & $\mathrm{x}$ & & & & & & & & \\
\hline & Marupa & Simarouba amara Aublet & SIMAROUBACEAE & & $\mathrm{x}$ & & & & $\mathrm{x}($ corteza $)$ & & $\mathrm{x}$ & \\
\hline & Picho huayo caspi & Siparuna guianensis Aublet & MONIMIACEAE & & & & & & & & & $\mathrm{x}$ \\
\hline & Cepanchina & Sloanea fragans Rusby & ELAEOCARPACEAE & $\mathrm{x}$ & & & & & & & & \\
\hline & Cepanchina & Sloanea grandiflora Smith & ELAEOCARPACEAE & $\mathrm{x}$ & & & & & & & & \\
\hline & Cepanchina & Sloanea guianensis (Aubl.) Benth. & ELAEOCARPACEAE & $\mathrm{x}$ & & & & & & & & \\
\hline & Cepanchina & Sloanea latifolia (Richard) Schumann & ELAEOCARPACEAE & $\mathrm{x}$ & & & & & & & & \\
\hline & Cepanchina & Sloanea multiflora Kosterm & ELAEOCARPACEAE & $\mathrm{x}$ & & & & & & & & \\
\hline & Cepanchina & Sloanea tuerckheimii J. D. Smith & ELAEOCARPACEAE & & & & & & & & & $\mathrm{x}$ \\
\hline & Casha pona & Socratea exorrhriza C. Mart & ARECACEAE & $\mathrm{x}$ & & & $\mathrm{x}$ & x(raíz) & x(raíz, chonta) & & & $\mathrm{x}$ \\
\hline & Ubos & Spondias mombin $\mathrm{L}$. & ANACARDIACEAE & & & $\mathrm{x}$ (personas $\mathrm{y}$ fauna) & & & $\mathrm{x}($ corteza $)$ & & & \\
\hline & Huarmi caspi & Sterculia apetala var apetala & STERCULIACEAE & & & & & & & & & $\mathrm{x}$ \\
\hline & Sacha cumaceba, chontaquiro & Swartzia cardiosperma Spruce ex Bentham & FABACEAE & & $\mathrm{x}$ & & & & $\mathrm{x}$ (madera) & & & $\mathrm{x}$ \\
\hline
\end{tabular}




\begin{tabular}{|c|c|c|c|c|c|c|c|c|c|c|c|c|}
\hline \multirow[b]{3}{*}{ No } & \multirow[b]{3}{*}{ NOMBRE COMÚN } & \multirow[b]{3}{*}{ NOMBRE CIENTÍFICO } & \multirow[b]{3}{*}{ FAMILIA } & \multicolumn{7}{|c|}{ ESPECIES DE IMPORTANCIA ECONÓMICA Y CIENTÍFICA } & \multirow{3}{*}{$\begin{array}{c}\text { ESPECIES } \\
\text { RARAS O DE } \\
\text { IMPORTANCIA }\end{array}$} & \multirow{3}{*}{$\begin{array}{c}\text { ESPECIES } \\
\text { RARAS O DE } \\
\text { DISTRIBUCIÓN } \\
\text { RESTRINGIDA } \\
\end{array}$} \\
\hline & & & & \multicolumn{7}{|c|}{ USOS LOCALES } & & \\
\hline & & & & \begin{tabular}{|c|} 
MADERA \\
REDONDA \\
\end{tabular} & \begin{tabular}{|c|} 
MADERA \\
ASERRADA \\
\end{tabular} & $\begin{array}{c}\text { USO } \\
\text { ALIMENTARIO } \\
\end{array}$ & $\begin{array}{c}\text { USO } \\
\text { ORNAMENTAL }\end{array}$ & \begin{tabular}{|l|} 
ARTESANÍA \\
INDUSTRIAL \\
\end{tabular} & MEDICINAL & USO & & \\
\hline \multicolumn{2}{|c|}{215 Sacha cumaceba } & Swartzia polyphylla DC. & FABACEAE & & & & & & $\mathrm{x}$ (madera) & $\mathrm{x}$ (madera) & $\mathrm{x}$ & \\
\hline \multirow{2}{*}{\multicolumn{2}{|c|}{$\begin{array}{l}216 \text { Remo caspi } \\
217 \text { Caoba }\end{array}$}} & Swartzia racemosa Benth & FABACEAE & $\mathrm{x}$ & & & & x(raíz y madera) & $\mathrm{x}($ corteza) & & & $\mathrm{x}$ \\
\hline & & Swietenia macrophylla King & MIMOSACEAE & & $\mathrm{x}$ & & & & & & & $\mathrm{x}$ \\
\hline \multicolumn{2}{|c|}{218 Azufre caspi } & Symphonia globulifera $\mathrm{L}$. & CLUSIACEAE & $\mathrm{x}$ & & $\mathrm{x}$ (fauna) & $\mathrm{x}$ & & $\mathrm{x}($ latex) & & & \\
\hline \multicolumn{2}{|c|}{219 Tangarana negra } & Tachigali melinonii (Harms) Z \& H & FABACEAE & & & & & & & & $\mathrm{x}$ & \\
\hline \multicolumn{2}{|c|}{220 Tangarana } & Tachigali paniculata Aublet & FABACEAE & & & & & & & & $\mathrm{x}$ & $\mathrm{x}$ \\
\hline \multicolumn{2}{|c|}{221 Tangarana } & Tachigali polyphilla & FABACEAE & & & & & & & & $\mathrm{x}$ & \\
\hline \multicolumn{2}{|c|}{222 Tangarana del bajo } & Tachigalia tessmannii Harms. Notizbl. & LEGUMINOSAE & $\mathrm{x}$ & & & & & & & & \\
\hline \multicolumn{2}{|c|}{223 Wuira caspi } & Tapirira guianensis Aublet & ANACARDIACEAE & $\mathrm{x}$ & $\mathrm{x}$ & & & & & & $\mathrm{x}$ & \\
\hline \multicolumn{2}{|c|}{224 Wuira caspi } & Tapirira retusa Ducke & ANACARDIACEAE & $\mathrm{x}$ & $\mathrm{x}$ & & & & & & & \\
\hline \multicolumn{2}{|c|}{225 Yacushapana } & Terminalia amazonia (J.F.G.) Exell & COMBRETACEAE & & & & & & & & & $\mathrm{x}$ \\
\hline \multicolumn{2}{|c|}{226 Huacapu negro } & Tetrastylidium peruvianum Sleumer & OLACACEAE & $\mathrm{x}$ & & & & & & & & \\
\hline \multicolumn{2}{|c|}{227 Macambo } & Theobroma bicolor Humb & STERCULIACEAE & & & $\mathrm{x}$ (personas $\mathrm{y}$ fauna) & & & & $x$ (semilla) & & \\
\hline & Sacha cacao & Theobroma obovatum Klo. ex. Ber. & STERCULIACEAE & & & $\mathrm{x}$ (personas $\mathrm{y}$ fauna) & & & & & & $\mathrm{x}$ \\
\hline & Sacha cacao & Theobroma subincanum C. Martius & STERCULIACEAE & & & $\mathrm{x}$ (personas $\mathrm{y}$ fauna) & & & & & & \\
\hline & Clavohuasca & Tynnanthus panurensis (Burt) Sandw & BIGNONIACEAE & & & & & $\mathrm{x}($ tallo $)$ & $\mathrm{x}$ (corteza) & $\mathrm{x}($ aceite $)$ & & \\
\hline & Pucacuro caspi & Toccoca guianensis Aubl. & MELASTOMATACEAE & & & & & & $\mathrm{x}$ (hojas) & & & \\
\hline & Chullachaqui caspi & Tovomita umbellata Bentham ex Engler & CLUSIACEAE & & & & & & & & & $\mathrm{x}$ \\
\hline & Copal & Trattinnickia aspera (Standley) Swart & BURSERACEAE & $\mathrm{x}$ & $\mathrm{x}$ & & & & & & & \\
\hline & Requia negra & Trichilia maynasiana C. DC. & MELIACEAE & & & & & & & & & $\mathrm{x}$ \\
\hline & Requia negra & Trichilia micrantha Bentham & MELIACEAE & & & & & & & & & $\mathrm{x}$ \\
\hline & Requia & Trichilia pleeana (Adr. Jussieu) C.DC. & MELIACEAE & $\mathrm{x}$ & $\mathrm{x}$ & & & & & & & \\
\hline & Requia & Trichilia septentrionalis C.DC. & MELIACEAE & $\mathrm{x}$ & $\mathrm{x}$ & & & & & & & $\mathrm{x}$ \\
\hline & Uña de gato & Uncaria guianensis (Aubl) Gmel & RUBIACEAE & & & & & & $\mathrm{x}$ (liana) & $\mathrm{x}$ (liana) & & \\
\hline & Carahuasca & Unonopsis floribunda Diels & ANNONACEAE & & & & & & & & & $\mathrm{x}$ \\
\hline & Cumalilla & Virola albidiflora Ducke & MYRISTICACEAE & & & & & & & & & $\mathrm{x}$ \\
\hline & Cumala negra & Virola caducifolia $\mathrm{W}$. Rodrigues & MYRISTICACEAE & & & & & & & & & $\mathrm{x}$ \\
\hline & Cumala blanca & Virola calophylla Warburg & MYRISTICACEAE & $\mathrm{x}$ & $\mathrm{x}$ & & & & & & & \\
\hline & Cumala & Virola decorticans Ducke & MYRISTICACEAE & $\mathrm{x}$ & $\mathrm{x}$ & & & & & & & \\
\hline & Cumala blanca & Virola duckei A.C. Smith & MYRISTICACEAE & $\mathrm{x}$ & $\mathrm{x}$ & & & & & & & \\
\hline & Cumala blanca & Virola elongata (Bentham) Warburg. & MYRISTICACEAE & $\mathrm{x}$ & $\mathrm{x}$ & & & & $\mathrm{x}($ latex) & & $\mathrm{x}$ & \\
\hline & Cumala negra & Virola multinervia Ducke & MYRISTICACEAE & & $\mathrm{x}$ & & & & & & & \\
\hline & Cumala & Virola obovata Ducke & MYRISTICACEAE & $\mathrm{x}$ & $\mathrm{x}$ & & & & & & & \\
\hline & Caupuri & Virola pavonis (D.C.) A.C. Smith & MYRISTICACEAE & & $\mathrm{x}$ & & & & $\mathrm{x}($ latex) & & & $\mathrm{x}$ \\
\hline & Cumala & Virola peruviana (A. DC.) Warburg & MYRISTICACEAE & $\mathrm{x}$ & $\mathrm{x}$ & & & & & & & \\
\hline & Cumala & Virola sebifera Aublet & MYRISTICACEAE & $\mathrm{x}$ & $\mathrm{x}$ & & & & & & & \\
\hline & Cumala, cumala blanca & Virola surinamensis (Rod) Warb. & MYRISTICACEAE & & $\mathrm{x}$ & & & & & & & \\
\hline & Pichirina & Vismia angusta Aublet & CLUSIACEAE & & & & & & $\mathrm{x}$ (savia) & & & \\
\hline & Pali perro & Vitex orinocensis var multiflora (Miq.) $\mathrm{H}$ & HuberVERBENACEAE & & & & & & & & & $\mathrm{x}$ \\
\hline & Quillosisa & Vochysia vismiifolia Spruce ex Warming. & VOCHYSIACEAE & $\mathrm{x}$ & $\mathrm{x}$ & & $\mathrm{x}$ & & & & & \\
\hline & Espintana & Xylopia parviflora Spruce & ANNONACEAE & $\mathrm{x}$ & & & $\mathrm{x}$ & & & & & \\
\hline & Espintana & Xylopia poeppiggiana R.E. Fries & ANNONACEAE & $\mathrm{x}$ & & & $\mathrm{x}$ & & & & & \\
\hline
\end{tabular}

